\title{
Criminologie
}

\section{Nouvelles avenues considérées par le service de probation}

\section{Michel Jacques Trottier, Marie-France Dufour, Jocelyne Amesse-Girard, Michel Lacoste et Robert Jacques}

Volume 12, numéro 2, 1979

Probation : aide ou contrainte ?

URI : https://id.erudit.org/iderudit/017107ar

DOI : https://doi.org/10.7202/017107ar

Aller au sommaire du numéro

Éditeur(s)

Les Presses de l'Université de Montréal

ISSN

0316-0041 (imprimé)

1492-1367 (numérique)

Découvrir la revue

Citer cet article

Trottier, M. J., Dufour, M.-F., Amesse-Girard, J., Lacoste, M. \& Jacques, R. (1979). Nouvelles avenues considérées par le service de probation. Criminologie, 12(2), 41-57. https://doi.org/10.7202/017107ar d'utilisation que vous pouvez consulter en ligne. 
NOUVELLES AVENUES CONSIDEREES PAR LE SERVICE DE PROBATION

\author{
Michel Jacques Trottier * \\ Marie-France Dufour \\ Jocelyne Amesse-Girard \\ Michel Lacoste \\ Robert Jacques
}

Trois aspects de l'horizon qui peut s'ouvrir au service de Probation ont été développés à titre d'essai. Parmi eux, la déjudiciarisation et la restitution sont déjà connues du monde correctionnel et même du grand public alors que l'autre, la mise en liberté provisoire, tel que présenté est particulièrement nouveau et même audacieux. L'ensemble des trois dimensions que comprend le présent article démontre l'intérêt que le service de Probation porte au développement des avenues originales en matière correctionnelle. $\AA$ l'heure actuelle, il est encore trop tôt pour s'engager concrètement dans ces voies, mais les idées développées ici sont des perspectives d'avenir.

\title{
I. LA DEJUDICIARISATION
}

La déjudiciarisation est un concept nouveau que la Commission de reforme du droit du Canada a mis en lumière au cour de ses travaux. Il a pour principaux aspects la prise en charge des délinquants par la collectivité, l'emploi de la discrétion policière en matière de poursuite afin de favoriser le règlement des conflits hors cour (conciliation) et finalement, en dernier ressort, l'application de mesures alternatives à l'emprisonnement utilisées par la cour au moment de disposer du judiciable.

Les autorités commencent à se rendre compte que le recours automatique au processus judiciaire, avec ses étapes et

* Michel Jacques Trottier : M.Sc., L.LL, agent de probation à Montreal, secteur Nord ;

Marie-France Dufour et Jocelyne Amesse-Girard : B.Sc., agents de probation à Montréal, secteur Sud ;

Michel Lacoste: B.Sc., agent de probation, responsable du bureau de Saint-Hyacinthe; Hyacinthe.

Robert Jacques : B.Sc., agent de probation du bureau de Saint- 
ses retards, surcharge nos cours de justice criminelle et aboutit souvent à l'inverse du résultat recherché par le système. C'est donc pour redonner à l'appareil judiciaire toute son efficacité que les gouvernements manifestent de plus en plus d'intérêt à l'égard de ces mesures alternatives. C'est aussi pour des questions de coût que l'État les envisage, car il semble bien que cet aspect, sans être primordial, n'est pas sans intérêt pour la communauté.

\section{A. Études des différents palliers d'interventions}

L'appellation \& déjudiciarisation 》 désigne les incidents réglés à l'intérieur de la collectivité, les affaires non transmises à la Justice par la police, les conciliations intervenues avant procès ou l'imposition de peines autres que les peines d'emprisonnement. Toutefois même si la notion elle-même va plus loin, nous nous limiterons, à dessein, dans cet article, à des aspects de la déjudiciarisation qui traitent des incidents réglés à l'intérieur de la collectivité ou des conciliations intervenues avant le procès. Nous excluons donc délibérément toutes les peines autres que les peines d'emprisonnement car cela nous obligerait à traiter de la Loi de la Probation plus particulièrement et des mesures prévues à l'article 662.1 du Code criminel déjà décrites dans un article précédent.

\section{La collectivité}

La première étape de la déjudiciarisation consiste dans le recours à la collectivité pour régler certains problèmes d'ordre social et moral sans avoir besoin nécessairement de recourir à la Justice. On en revient donc dans une certaine mesure à la Justice privée sans qu'on doive pour cela penser à un retour à une justice primitive.

Actuellement ce recours à la collectivité existe pour de nombreux organismes tels que les corporations professionnelles, les entreprises pour leur propre service de sécurité et les grands ensembles d'habitation qui peuvent régler un certain nombre de problèmes par eux-mêmes sans avoir recours à la Justice pénale. La police fait aussi à l'occasion appel à la collectivité pour éviter à certaines catégories de délinquants des poursuites criminelles. 


\section{La police}

Elle dispose d'un pouvoir de discrétion qu'elle utilise et qu'elle a toujours utilisé en particulier à l'égard de jeunes délinquants. Dans d'autres cas, elle fait aussi usage de sa discrétion pour les délits tels que la conduite en état d'ivresse ou sous l'effet de la drogue, ou encore la conduite criminelle en raison d'une maladie mentale.

Cette discrétion, laissée à la police, de se référer ou non aux autorités judiciaires, pose le problème de l'égalité de tous devant la Justice. Le problème est plus aigu depuis l'adoption par le gouvernement canadien de la Déclaration canadienne des droits de l'homme et de la Charte québécoise des droits de la personne par le gouvernement du Québec.

Il apparaît, dès lors, très important d'établir des critères précis et non équivoques pour maintenir le droit de chacun à une justice impartiale. La Commission de réforme du droit ${ }^{1}$ dans l'un de ses documents met de l'avant un certain nombre d'éléments dont on pourrait se servir pour déterminer s'il y aura inculpation ou non; la gravité de l'infraction est-elle de nature à exiger un procès dans l'intérêt public, la collectivité est-elle en mesure de supporter les conséquences du classement de l'affaire, existe-t-il d'autres moyens efficaces de traitement de l'affaire pour qu'elle ne se reproduise plus, les conséquences de l'arrestation ou des poursuites ne seront-elles pas trop lourdes pour l'inculpé ou sa famille en regard du délit commis, la vietime et le délinquant sont-ils favorables à un arrangement à l'amiable ?

Ces recommandations de la Commission constituent cependant un aspect nouveau étant donné que ce n'est plus une discrétion réelle laissée à la police mais une discrétion éclairée, exercée selon des critères précis et des politiques connues de tous. Il reste cependant à appliquer une telle politique de façon cohérente malgré la disparité des corps policiers. Comment assurer une interprétation uniforme et cohérente? La réponse est loin d'être donnźe puisque actuellement tout le système policier est apprécié principalement quant à son efficacité par rapport au nombre d'affaires résolues où il en résulte une arrestation, une inculpation et finalement une condamnation.

1. La Déjudiciarisation, Commission de la réforme du droit du Canada, document de travail no 7 , p. 8. 


\section{La conciliation avant procès}

La dénonciation étant le point de départ de la poursuite en matière pénale, c'est donc au procureur général, par l'entremise de ses substituts, d'engager ces poursuites.

En pratique cependant c'est à la police qu'il appartient dans chaque cas de poursuivre et de décider sous quel chef elle va le faire. En effet le procureur de la Couronne s'en remet aux policiers pour prendre dans la majorité des cas les décisions courantes d'inculper les justiciables. Pour les actes criminels plus graves c'est le poursuivant qui détient le pouvoir discrétionnaire de choisir les cas où il va intenter des poursuites, de déterminer le chef d'inculpation, et de modifier ou de retirer certaines inculpations.

C'est donc ici que peuvent jouer les règlements de conciliation proposés par la Commission et qui incitent à faire usage de son pouvoir discrétionnaire dans le choix des affaires qui doivent être soumises à l'appréciation des tribunaux. Car même si une inculpation est portée, est-il vraiment utile que le processus soit entamé et passe par toutes les phases du procès jusqu'à un verdict de culpabilité et de la condamnation ? Est-il possible de traiter par voie de conciliation un certain nombre d'affaires?

Encore là, il s'avèrera important de définir clairement les cas pour lesquels une conciliation s'impose de préférence à un procès sur la définition des critères que devraient suivre les procureurs de la poursuite dans leur décision de procéder plutôt que de recourir à la conciliation.

Ces critères ne devraient pas se limiter à certaines infractions et ne devraient pas nécessairement exclure les délits où il $\mathrm{y}$ a violence. C'est notamment dans ces cas où les victimes et les accusés se connaissent que la conciliation a le plus de chance de se révéler efficace car le délit peut résulter de divers facteurs tels que l'alcool, la drogue, etc. Cette politique devrait cependant exclure la conciliation dans les cas de meurtre, de viol ou de vol à main armée car il y a des situations où l'intérêt public doit primer sur les intérêt particuliers.

Enfin, cette position de conciliation devrait viser à ce que les décisions de conciliation soient prises au grand jour et répon- 
dent également à des critères conformes à ceux qui seraient exigés pour l'exercice du pouvoir discrétionnaire de la police et celui du poursuivant.

La Commission de réforme du droit soumet à ce stade un certain nombre de critères tenant compte de l'intérêt de la victime et de la collectivité et met de l'avant plus particulièrement les facteurs suivants :
a) l'affaire qui fait l'objet de l'enquête peut-elle être classée par la police ;
b) les faits en cause sont-ils suffisamment graves pour jus-
tifier des poursuites et les preuves sont-elles suffisantes;
c) est-il possible d'affirmer que la victime et l'inculpé se connaissaient avant les faits ;
d) les faits en l'espèce sont-ils contestés ;
e) l'inculpé et la victime sont-ils d'accord pour accepter une conciliation au lieu de recourir à un procès ;
f) les prérogatives et les intérêts de la société, du délin- quant et de la victime sont-ils mieux servis par le moyen d'une conciliation que par l'intermédiaire d'un verdict de culpabilité et d'une condamnation ;
g) un procès et une condamnation sont-ils susceptibles de produire un préjudice injustifié à sa famille ou d'aggra- ver les problèmes sociaux qui sont à l'origine de son acte criminel ${ }^{2}$.

Il convient finalement de faire remarquer que l'application de l'un de ces critères ne peut affecter le pouvoir de la poursuite de retirer une inculpation comme elle en a le droit, ou d'interdire à la Cour de prononcer un non lieu si la poursuite reprend l'accusation, ou de suggérer une mise en liberté conditionnelle ou inconditionnelle.

Il demeure par ailleurs évident dans notre esprit qu'il faudra également répondre à un certain nombre de questions avant de généraliser une telle mesure. On pense ici plus précisément au consentement de toutes les parties impliquées, au type de consentement auquel il faut s'attarder, à la nécessité de procéder à une inculpation en bonne et due forme, à la question de savoir si le délinquant devra obligatoirement avoir reconnu sa responsabilité, aux règles de preuve s'appliquant au statut du délin- 
quant face à la loi du casier judiciaire. Autant de points à examiner avant de se situer définitivement face à une telle mesure.

\section{LA DÉJUdICIARISATION ET LE SERVICE DE PROBATION}

La déjudiciarisation nous permet ainsi d'envisager les actes des contrevenants sous une nouvelle dimension à la fois sociale et psychologique. Une telle approche, dans la mesure où elle peut être discriminatoire peut être critiquée par les tenants d'une justice égale pour tous. Il faudra de ce fait, voir à ce que les règles du jeu soient clairement définies surtout vis-à-vis les substituts du procureur général du Québec qui se montrent très réticents, puisque cette mesure implique une remise en question partielle de leur rôle traditionnel.

On peut alors se demander si le service de Probation à titre d'organisme gouvernemental faisant partie de l'appareil judiciaire ne constituerait pas la charnière idéale permettant le dialogue entre les parties en vue de l'établissement d'une telle mesure.

Le processus de déjudiciarisation exigeant une phase d'évaluation, il paraît normal que le service de Probation y soit impliqué compte tenu de l'expérience acquise au plan présentenciel. Dès que la police ou la poursuite auront opté pour une forme de déjudiciarisation, le service de Probation pourrait être appelé à fournir une évaluation psycho-sociale qui mesurerait le bienfondé de la décision préconisée en fonction de critères déterminés au préalable. Suite à une décision positive, l'agent de probation verrait à coordonner les diverses modalités de l'entente et à favoriser, le cas échéant, la conciliation entre la victime et le contrevenant.

Le processus décrit plus haut aurait l'avantage de suggérer un cadre d'assistance individualisée tout en laissant aux policiers ou à la poursuite l'élément décisionnel quant au recours à une telle mesure.

De cette façon on faciliterait un règlement rapide d'un conflit à la satisfaction de la victime tout en incitant le délinquant à prendre ses responsabilités.

À titre de remarques finales, on pourrait souhaiter que le législateur suive la voie préconisée par la Commission de réforme du droit du Canada et adopte les amendements au Code criminel permettant le recours à la déjudiciarisation. En déterminant 
rigoureusement les types de personne pouvant bénéficier de cette mesure et en codifiant les critères objectifs qui devront être suivis par les différents niveaux chargés de l'application du code pénal, on favoriserait alors une meilleure utilisation de la discrétion qui appartient à la fois à la police et au poursuivant.

\section{LA MISE EN LIBERTÉ PROVISOIRE}

Toujours dans une optique d'élargissement de nos sphères d'intervention, il nous apparaît pertinent de considérer l'éventualité de l'implication du service de Probation au niveau de la mise en liberté provisoire. Cet intérêt nouveau provient du fait que pour plusieurs agents de probation, notre action doit de plus en plus viser une meilleure intégration de l'individu et son mieux-être au sein de la collectivité. Touchant directement la population aux prises avec des démêlés judiciaires, c'est évidemment celle-ci que notre service cherche à atteindre, d'autant plus qu'il est présentement le principal, sinon le seul service à dimension sociale à la portée de plusieurs tribunaux. Dans cette ligne de pensée, nous ne pouvons négliger les divers problèmes qu'on retrouve très souvent chez les individus traduits en cour, problèmes d'autant plus importants qu'ils sont parfois à la source de leurs ennuis légaux. On peut même remarquer que pour plusieurs de ces personnes, les ressources environnantes sont inconnues ou inexistantes et en ce sens, leur comparution devant les tribunaux peut représenter l'occasion d'intervenir au moment opportun. Or, notre expérience démontre que, très souvent, notre intervention auprès d'un justiciable survient beaucoup trop tardivement, c'est-à-dire lorsque la situation s'avère passablement détériorée. Ce constat et la recherche d'une action plus profitable nous ont incités à explorer le domaine de la mise en liberté provisoire et à l'analyser succinctement dans les pages qui suivent.

Après avoir défini brièvement en quoi consiste cette mesure, nous tenterons d'évaluer jusqu'à quel point notre action pourrait être possible et souhaitable. Dans cette démarche, nous tiendrons compte des diverses étapes du processus judiciaire, et de ce qui nous apparaîtrait réalisable et bénéfique pour le justiciable.

\section{A. Contexte légal}

Tout d'abord, il est sans doute utile de rappeler que, selon l'esprit de la loi, la mise en liberté provisoire doit être accordée 
à un justiciable, à moins que le poursuivant ne fasse valoir des motifs justifiant la détention (art. 457 (1) et 457 (5) C.cr.). Les critères d'octroi ou de refus d'un cautionnement sont clairement spécifiés à l'article 457 (7) C.cr.; il s'agit d'abord de s'assurer de la présence ultérieure du prévenu au tribunal et, en deuxième lieu, de garantir l'intérêt, la protection et la sécurité du public. De plus, lorsqu'il libère un individu sous cautionnement, le juge peut imposer diverses conditions, entre autres, celle de se présenter à un agent de la paix ou à toute autre personne désignée (art. 457 (4) a) C.cr.). Jusqu'à présent, on peut dire que, de façon générale, les personnes à qui on imposait une telle condition, devaient se rapporter au poste de police selon une fréquence déterminée.

\section{B. Position actuelle du service de Probation}

Par ailleurs, il est arrivé occasionnellement, surtout après une déclaration de culpabilité, que les juges nous confient certains cas particuliers. D'après notre expérience personnelle, nous avons pu constater que, la plupart du temps, il s'agissait d'individus nécessitant une intervention urgente (problèmes d'hébergement, financiers, psychiatriques) ou dont le juge voulait évaluer le comportement pendant une période donnée. Ce furent là des cas exceptionnels puisque de façon générale le service de Probation a toujours évité jusqu'ici de superviser des individus qui ne lui étaient pas référés en vertu des dispositions des articles 662, 663 ou 665 du Code criminel. C'est ainsi que, de par ses deux rôles traditionnels axés sur la probation et l'évaluation présentencielle, le service de Probation a centré son action auprès des justiciables dont la reconnaissance de culpabilité avait été enregistrée. C'est donc dire que si, en vertu de l'article 457 (4) a) du Code criminel, le service de Probation décidait de s'impliquer lors d'une mise en liberté provisoire, il étendrait du même coup son champ d'action en s'intéressant à tout prévenu ou accusé, et ce dès les étapes préliminaires du processus judiciaire.

C. Le pour et le contre d'une implication du service de Probation

Voyons maintenant de quelle façon et à quelle étape le service de Probation pourrait s'engager, compte tenu des aspects 
positifs et négatifs qui se dégageront de notre brève analyse. Dans cette démarche, on constatera que, dépendemment de l'étape où on se situe, l'intervention aura une portée socialisante, thérapeutique et/ou de contrôle.

\section{Avant la reconnaissance ou le plaidoyer de culpabilite}

Un individu référé à cette première étape du processus judiciaire pourrait recevoir deux types d'aide : une aide morale (possibilité de s'exprimer, d'être entendu, de dialoguer sur l'expérience vécue) dans le but de personnaliser et d'humaniser l'application de la justice, ou une aide sociale (hébergement, aide psychiatrique, financière, par l'entremise d'agences sociales, médicales, psychiatriques ou autres). Ces deux types d'aide constitueraient une approche ayant un objectif exclusivement thérapeutique.

Conformément à l'article 457.3 (1) C.cr., rien ne nous autoriserait alors à questionner le prévenu relativement à l'infraction dont il est inculpé. La loi actuelle ne nous protégeant aucunement au plan de la confidentialité, un intervenant qui s'impliquerait à ce niveau risquerait fort d'être contraint de témoigner en cour, ce qui, dès lors, l'éloignerait du but visé initialement.

Par ailleurs, une intervention qui, à l'intérieur d'une relation d'aide, excluant une dimension spécifique des problèmes d'un individu (délit actuel, antécédents, milieu criminalisé), offrirait certainement, de par sa nature même, de nombreuses limites.

Toutefois, dans l'optique où, à cette étape du processus judiciaire, la Cour nous confierait un individu dans le but principal d'exercer une forme de contrôle, nous serions alors tenu de rendre compte au tribunal du respect des conditions.

Cette nouvelle mesure souligne à nouveau la dualité traditionnelle du rôle d'agent de probation à savoir sa détermination à agir au sein d'une relation d'aide et son obligation à exercer un contrôle délégué par la Cour. On constate ainsi l'ambiguïté du rôle de l'intervenant qui, sans confirmation légale claire de son mandat, à cette étape des procédures, ne pourrait qu'osciller entre l'individu et ses besoins d'une part, et la justice et son application d'autre part. 


\section{Après la reconnaissance ou le plaidoyer de culpabilité}

La situation s'avère quelque peu différente à partir du moment où l'individu a plaidé coupable ou est reconnu comme tel. L'implication du service de Probation à cette étape présentencielle, peut être perçue de la même façon qu'au niveau précédent, à l'exception du fait qu'à ce stade des procédures la présomption d'innocence étant écartée, l'intervention peut être beaucoup plus globale et non pas seulement limitée comme précédemment à des problèmes spécifiques où on ignore forcément l'ensemble de la vie de l'individu. Cependant, il nous semble important ici de se montrer très vigilants face à une tendance qui, ayant déjà été manifestée lorsqu'on nous référait des cas de façon informelle, pourrait prendre beaucoup plus d'ampleur si le service s'engageait davantage dans un tel type de supervision. Il serait en effet très facile et tentant, en utilisant ou non le couvert de l'aide qu'on peut apporter, de nous confier des individus dans le seul but d'évaluer leur fonctionnement et évolution pendant une période donnée. De cette façon, on pourrait craindre, d'une part, des remises nombreuses, mettant ainsi en péril le droit de l'individu à recevoir sa sentence dans les plus brefs délais, et, d'autre part, des sentences qui tiendraient compte, non plus principalement de l'acte criminel reproché, mais bien d'une évolution de quelques mois, ce qui, par surcroît, diminuerait l'impact d'un appel pouvant être interjeté par les diverses parties. Selon nous, une telle pratique risquerait de fausser l'esprit de la justice, en amenant toutes sortes d'interprétations, attitudes et attentes selon les différentes personnes concernées, et selon l'importance que chacune d'entre elles accorde aux notions de contrôle et d'aide.

Les constatations précédentes et les dangers relevés s'appliquent également lorsque, suite à un rapport présentenciel à l'intérieur duquel un plan de traitement et un pronostic ont été soumis par l'agent, éléments que la Cour peut vouloir vérifier empiriquement en reportant la sentence à une date ultérieure et en nous confiant le sujet entre-temps. De cette façon, l'accusé vit ainsi une période de mise à l'essai plus ou moins significative et appropriée à sa personnalité, mais qui néanmoins, peut influencer fortement la sentence qui va suivre. 


\section{Préalables à considérer}

Une implication du service de Probation lors de la mise en liberté provisoire nécessiterait, d'abord, l'établissement de normes d'application.

On devrait, en premier lieu, déterminer l'orientation de la mesure. Devrait-elle être axée uniquement sur le contrôle, ou sur l'aspect thérapeutique, ou sur les deux dimensions à la fois ?

Dans l'éventualité où l'on opterait pour un contrôle exclusif, notre efficacité à ce niveau nous apparaîtrait dérisoire comparée à celle pouvant être appliquée par les corps policiers. En effet, alors que notre mesure de contrôle ne porterait que sur le respect de la condition de se présenter à nos bureaux, le contrôle policier offre plus de garanties, au sens où il assure un éventail plus grand de possibilités. De plus, il deviendrait superflu d'établir des critères de sélection des cas puisque nous serions dans l'obligation de les accepter tous.

Advenant que nous options pour une mesure incluant à la fois le contrôle et l'aide clinique, l'élaboration de critères de sélection des cas deviendrait primordiale. La définition de ces critères, aussi bien pour les juges que pour nous, devrait préalablement s'effectuer en vertu d'un consensus.

De plus, il faudrait s'entendre pour que l'agent de probation ne soit pas tenu de divulguer, dans une certaine mesure, les confidences du sujet. Ce point revêt une importance capitale surtout dans le cas où ce dernier nous serait référé au moment de la comparution.

Il y aurait lieu également de considérer quelle serait la façon la plus adéquate de rendre compte à la cour des démarches effectuées relativement à un cas qu'elle nous a référé et, forcément, des résultats de nos démarches. Ce faisant, il devient impossible de ne pas l'informer du type de collaboration offerte par le sujet.

Par ailleurs, dans l'optique où nous devrions répondre à des urgences, il faudrait certes établir des ententes avec les agences sociales appropriées, tant en matière d'hébergement, d'aide financière ou médicale.

Si notre action se veut exclusivement à portée sociale, les considérations retenues plus haut restent toujours valables, à 
cette exception près que le sujet ne serait plus tenu de se présenter au service de Probation. Il s'agirait plutôt d'implanter, auprès de la cour, un service de référence pouvant être utilisé par les justiciables en période de mise en liberté provisoire, nettement distinct du type de services que nous avons offerts jusqu'à présent.

Selon cette nouvelle perspective, que nous entrions en contact avec l'accusé à n'importe quelle étape du processus judiciaire n'a plus tellement d'importance. Toutefois, il apparait de beaucoup préférable d'intervenir rapidement, dès que le besoin est manifesté, donc autant que possible au moment de la comparution.

D'une manière générale, il convient donc de constater que le besoin d'aide auprès des justiciables est bien présent et qu'il réclame une voie de solution.

Le service de Probation serait sans doute en mesure d'intervenir dans plusieurs cas, tenant compte des préalables mentionnés antérieurement mais en premier lieu dans un but d'exploration.

Par ailleurs, d'autres organismes à vocation sociale, seraient susceptibles de combler avantageusement et plus globalement les divers besoins qu'on pourrait retrouver chez une telle population. En ce qui nous concerne nous sommes portés à privilégier l'implication de telles ressources du fait qu'elles ne sont pas généralement confondues avec l'appareil judiciaire.

\section{LA RESTTTUTION CORRECTIONNELLE}

Le concept de restitution, bien que relativement ancien, est mal connu en général. On confond souvent restitution, dédommagement et indemnisation. La Commission de réforme du droit distingue les concepts de dédommagement (ou restitution) et d'indemnisation :

Le terme dédommagement ne s'entend que lorsque le délinquant dédommage personnellement la victime de son délit. L'opération s'effectue entre le délinquant et la victime et revêt donc un caractère personnel. D'un autre côté, l'indemnisation couvre un caractère impersonnel et s'entend d'un dédommagment ou d'un paiement effectué par l'Etat au profit de la victime ${ }^{3}$.

3. La Commission de réforme du droit du Canada (1976). La participation communautaire à la réadaptation du délinquant, Ottawa, Canada. 
Le Code criminel canadien consacre quelques dispositions au dédommagement et à la restitution. L'article 653 du Code stipule qu'au moment du prononcé de la sentence, la victime peut demander au juge que lui soit payée par le délinquant une somme d'argent pour le dédommagement de la perte ou des dommages occasionnés à ses biens. Cependant, dans la pratique, l'application de cette disposition est peu invoquée.

D'autre part, la restitution imposée à l'intérieur d'une ordonnance de probation est prévue à l'article 663 (2) (e) et est utilisee plus fréquemment. Cette disposition se lit comme suit :

Faire restitution ou réparation à toute personne lésée ou blessée du fait de l'infraction, de la perte ou du dommage véritable soufferts de ce fait par cette personne 4.

C'est cette disposition qui intéressait surtout le service de Probation du Québec. Le 16 septembre 1976, ce dernier choisissait à titre d'objectif spécifique de raviver la restitution monétaire comme mode de "sentencing 》. A cet effet, un comité d'étude a été formé. Il avait pour tâche d'étudier cette mesure, de familiariser les agents de probation à celle-ci et d'en faire connaître les divers modes d'application.

En mars 1977, le ministre de la Justice rendait publique la promotion de cette mesure et il réitérait cet engagement au cours de la conférence sur la déjudiciarisation tenue à Québec, du 24 au 26 octobre 1977.

\section{A. L'utilisation actuelle de ce mode d'intervention}

Malgré la formation d'un comité d'étude et les engagements du ministère de la Justice, le service de Probation ne s'est que fort peu impliqué dans cette mesure jusqu'à maintenant. Tout de même, il utilisa l'opportunité que lui offrait le rapport présentenciel pour promouvoir directement l'utilisation de cette mesure par les cours. Également il fut amené à s'y impliquer d'une façon indirecte dans le cadre de l'application des ordonnances de probation qui comportaient une condition de a faire restitution , à la victime.

Lors de la préparation d'un rapport présentenciel, l'agent de probation peut estimer que la restitution est une mesure souhaitable et il en fait la recommandation à la cour dans son

4. Code criminel canadien. 
rapport. Avant d'en arriver à cette conclusion cependant, il se doit de faire deux démarches précises : il cherche d'abord à obtenir que l'accusé endosse personnellement la décision de dédommager sa victime pour les dommages causés ce qui peut résulter en une meilleure motivation du justiciable. L'impact du geste réparateur devient de ce fait plus positif en termes de réhabilitation. Dans un deuxième temps, il évalue la capacité de payer de ce dernier; ceci a pour but d'éviter que l'obligation ne se transforme pour le justiciable en une incapacité de faire face à ses obligations ou de pourvoir adéquatement aux besoins de sa famille. Une telle conséquence irait alors à l'encontre de lobjectif premier de réhabilitation que vise cette forme de " sentencing 》, surtout si, en plus, le contrevenant se voit subséquemment pénalisé par l'imposition d'une peine de détention pour manquement à son ordonnance.

La restitution dans le cadre d'une ordonnance de probation avec surveillance, sans qu'il n'y ait eu rapport présentenciel au préalable, permet à l'agent de probation une implication moindre dans l'application de cette mesure. Jusqu'à maintenant, dans ces cas, son rôle s'est limité à expliquer au justiciable cette condition particulière de l'ordonnance de probation. Occasionnellement, l'agent tente de mettre en relation la victime et le délinquant lorsqu'il pense que cela peut faciliter le dédommagement ou pour des fins de réhabilitation.

Actuellement, la suite du processus échappe en grande partie au service de Probation. Ainsi, c'est le président du tribunal qui seul évalue les montants à rembourser ainsi que les modalités et les délais de paiement. Il relève également de la cour d'identifier les victimes auxquelles doivent être faits ces remboursements. Cette façon de procéder n'est pas sans entraîner certaines conséquences malheureuses comme par exemple, l'absence d'uniformité dans le déroulement du processus de restitution et l'obtention de résultats inférieurs en termes de réhabilitation à ceux auxquels on serait en droit de s'attendre. Ainsi, une étude effectuée par le service de Probation en 1978 révèle que $21 \%$ des ordonnances de probation comportant une condition de restitution ne précisent aucunement le montant à rembourser. Dans $24 \%$ des cas, il n'y a aucune indication sur l'identité des personnes à qui le remboursement doit être effectué. Seul le délai de restitution est clairement défini dans tous les dossiers. 
De plus, les mécanismes de contrôle et de collection des sommes d'argent se doivent également d'être précisés. Une étude évaluative effectuée au Minnesota, à ce sujet, démontre clairement que lorsque l'agent de probation assume un rôle de collecteur, cela s'avère un échec. Il semble que cette situation entre en conflit avec le rôle premier de l'agent, soit celui de conseiller. L'étude rapporte que les greffes, comme agence de collection, connaissent un taux de succès relativement plus élevé de remboursement. Le service de Probation du Québec s'est attardé à cette seconde modalité. Cependant, les mécanismes nécessaires ne sont pas encore institués, ce qui crée certaines injustices. En effet, une des conclusions de l'étude mentionnée plus haut, indique que les greffes n'entreprennent des procédures que dans $54 \%$ des cas pour non-respect de la condition de restitution.

Jusqu'à présent, cette application de la mesure de restitution, bien que réservée, a permis au service de Probation d'élargir son champ d'action en tant que conseiller auprès de la cour et de rendre ainsi un meilleur service à la communauté en essayant de montrer une image de la justice plus humaine et plus près de la réalité quotidienne.

Cette approche, à n'en plus douter, demeure valable. Il existe cependant une autre façon d'envisager la restitution monétaire. C'est celle qui permettrait à la victime et au contrevenant de jouer un rôle plus important dans la solution d'une situation conflictuelle les concernant. Elle suppose également une implication plus intensive du service de Probation et ce, dès la reconnaissance du plaidoyer de culpabilité et avant le prononcé de la sentence. Il s'agit de la restitution correctionnelle.

\section{B. La restitution correctionnelle}

Ce concept qui tend avant tout à favoriser la conciliation entre l'accusé et la victime a déjà été longuement traité par Romine R. Deming dans la revue Federal Probation ${ }^{5}$. Considérant l'expérience vécue dans le district judiciaire de SaintHyacinthe d'avril 1977 à août 1978 et tenant compte d'une part de l'utilisation de la mesure de restitution par les tribunaux et d'autre part de la réserve manifestée par le service de Pro-

5. Romine R. Deming, - Correctional Restitution : A Strategy for Correctional Conflict Management , Federal Probation, September 1976. 
bation à l'égard d'une implication accrue dans ce mode de "sentencing , il apparaît souhaitable que le concept de restitution correctionnelle soit sérieusement considéré par ce service et que son application soit envisagée à court terme.

Le service de Probation possède un personnel expérimenté capable de mettre sur pied les mécanismes nécessaires pour faire de la restitution un instrument valable de réinsertion sociale appliquée. Ainsi pour ce faire, il faudra envisager une intervention de l'agent de probation dans chacun des dossiers où la cour, à cause de la nature même du délit, envisage la possibilité de rendre une ordonnance de restitution. Cette intervention pourrait avoir pour buts principaux d'informer le délinquant et la victime de la possibilité d'une telle forme de sentence ou complément de sentence et d'obtenir leur collaboration pour le déroulement futur du processus. Par la suite, l'agent de probation, la victime et l'accusé pourraient évaluer ensemble la capacité de payer de ce dernier, convenir des modalités de paiement et préciser les délais nécessaires.

Le contrat de restitution ainsi établi et accepté par les trois parties serait alors soumis à l'approbation du président du tribunal qui, en l'acceptant, lui donnerait une valeur significative en l'incorporant à une ordonnance de probation, soit comme mesure unique ou soit comme complément d'une sentence.

Ainsi, l'agent de probation pourrait jouer pleinement son rôle de conseiller auprès de la cour et d'agent de réinsertion sociale. En effet, par son intervention au début du processus de * sentencing > il se sera acquis la collaboration de la victime et du justiciable et pourra plus aisément soumettre cette éventualité de la restitution au tribunal.

La restitution correctionnelle a des avantages marqués sur la simple indemnisation car le but recherché par la première est avant tout le rétablissement de la relation entre la victime et le délinquant, telle qu'elle existait avant le délit. De plus, cette modalité de réinsertion sociale permettrait à la victime de percevoir son agresseur 》 avec plus de réalisme diminuant ainsi l'impact des préjugés et des stéréotypes connus; également, cette approche permettrait au justiciable de prendre conscience du tort véritablement subi par la victime du fait de la commission de son délit. 
Il va de soi que l'implication d'un programme structuré de restitution monétaire ou correctionnelle nécessite des efforts de collaboration et de coordination des sous-systèmes impliqués. Une clarification des objectifs d'une telle mesure suivie d'une consultation générale des parties impliquées (tribunaux, greffes, service de Probation) devraient précéder la décision d'accroître ou non l'implication des agents de probation à l'égard de cette forme particulière de esentencing 》. Le besoin relatif à cette forme de sentence a été clairement identifié par la Commission de réforme du droit du Canada et il ne peut plus être mis en doute. L'étude doit maintenant porter sur les modalités d'application.

Les trois dimensions de l'intervention, traitées dans cet article, démontrent que l'agent de probation peut d'ores et déjà entrevoir dans ces nouvelles avenues un enrichissement de son agir professionnel. Dans certains cas, plusieurs questions préalables devront recevoir une réponse avant qu'il ne soit permis de croire en la viabilité du projet. L'expérience sur les sentences de travaux communautaires démontre qu'avec une préparation adéquate de nouveaux concepts peuvent efaire leur chemin s. Il est à souhaiter que l'on puisse fournir la même opportunité aux nouvelles idées que l'on vient de présenter. 